\title{
Evaluation of ocular findings in patients with lichen planus
}

\author{
Emin Ozlu¹, Kuddusi Teberik ${ }^{2}$
}

1Department of Dermatology, School of Medicine, Duzce University, Duzce, Turkey

2Department of Ophthalmology, School of Medicine, Duzce University, Duzce, Turkey

Adv Dermatol Allergol 2019; XXXVI (3): 267-271

DOI: https://doi.org/10.5114/ada.2018.74464

\begin{abstract}
Introduction: Lichen planus (LP) is a chronic, inflammatory disease of unknown etiology.

Aim: To evaluate the tear functions and the retinal and choroidal thickness (CT) in patients with LP.

Material and methods: In total, 33 patients and 30 healthy controls were enrolled. All participants were evaluated using the Ocular Surface Disease Index (OSDI) questionnaire, Schirmer 1 test (with anesthesia), tear break-up time (TBUT) test, intraocular pressure (IOP), axial length (AL), and central corneal thickness (CCT). The measurements of retinal thickness (RT) and CT were obtained by spectral domain optical coherence tomography.

Results: No significant difference was observed between the groups in IOP, AL, CCT, or RT ( $p>0.05$ in each group). There was a significant difference in the Schirmer 1 test, TBUT, and OSDI questionnaire ( $p<0.05$ in each group). Choroidal thickness in LP patients was thinner than that in the controls ( $p=0.001$ in each location). There was a moderate negative correlation between the disease duration and Schirmer 1 test, TBUT, and subfoveal CT $(r=-0.426, r=-0.555, r=-0.637 ; p=0.001, p=0.001, p=0.001$, respectively). There was a moderate negative correlation between the oral mucosal involvement and Schirmer 1 test, TBUT, and subfoveal CT $(r=-0.345, r=-0.392$, $r=-0.467 ; p=0.006, p=0.001, p=0.001$, respectively). There was a positive correlation between disease duration, oral mucosal involvement and OSDI score $(r=0.717, r=0.345 ; p=0.001, p=0.006$, respectively).

Conclusions: Lichen planus may influence tear function tests and may cause dry eye. Patients with LP had lower CT values than healthy controls. Further studies are needed to clarify the effect of LP on the eyes.
\end{abstract}

Key words: cornea, choroid, eye, lichen planus, tears.

\section{Introduction}

Lichen planus (LP) is a chronic inflammatory disease with mucocutaneous involvement. Etiology and pathogenesis of the disease are still not completely known yet [1]. Skin and mucosal involvement is seen together in $40 \%$ of patients with LP [2]. Mucosal involvement is more chronic, with a more severe course [3]. Lichen planus may involve ocular structures, although rarely, and the ocular involvement is combined with skin or oral involvement in general [4]. The disease may be manifested by eyelid and corneal involvement or cicatricial conjunctivitis [2-4]. Blepharitis, keratitis, symblepharon, corneal ulceration and residual visual impairment may develop due to conjunctival involvement [4]. In addition, LP may cause lacrimal canal pathologies and epiphora $[5,6]$. The number of case-control studies about the effects of $L P$ on the eye is quite limited up to now [7].
Eye involvement may also be seen in psoriasis, in which a physiopathological process similar to LP in terms of chronic inflammation is observed [8]. Especially inflammatory ocular changes and ocular surface diseases have been reported to occur in psoriasis [9]. Studies on the ocular involvement in psoriasis have been especially focused on the anterior segment diseases of the eye $[10,11]$. However, the posterior segment structures may also be affected in diseases with a chronic inflammatory course, and evaluations should include the posterior segment structures of the eye [12]. Current studies have demonstrated that psoriasis may affect the posterior segment structures of the eye $[8,13]$. To our knowledge, no study has evaluated the posterior structures of the eye in LP. Also, the number of case-control studies on the effects of LP on the anterior segment of the eye is quite limited.

Address for correspondence: Assist. Prof. Emin Ozlu MD, Department of Dermatology, Faculty of Medicine, Duzce University, 81200 Duzce, Turkey, phone: +90 (505) 2788174, fax: +90 3805421128, e-mail: dermatologg@gmail.com Received: 16.02.2018, accepted: 22.02.2018. 


\section{Aim}

This study aimed to evaluate the tear function tests, the Ocular Surface Disease Index (OSDI) questionnaire, intraocular pressure (IOP), axial length (AL), central corneal thickness (CCT), retinal thickness (RT) and choroidal thickness (CT) in LP, which is a chronic inflammatory disease, and to determine the changes in both anterior and posterior segments and compare them with the control group.

\section{Material and methods}

\section{Study design and patients}

This was a prospective controlled study, which was conducted in the light of the Declaration of Helsinki and followed a protocol approved by an institutional ethical review board. Patients were recruited in the study after being given informed consent. We included 33 patients with LP and 30 healthy controls in this study. All patients were obtained from a dermatology outpatient clinic. Patients aged over 18 years and whose diagnosis was confirmed by clinical and histopathological findings were included. The inclusion criteria for all subjects were best corrected visual acuity of 20/20 or more and refractive errors between $+1 \mathrm{D}$ and $-1 \mathrm{D}$ spherical equivalent. $\mathrm{Pa}-$ tients with prior use of ultraviolet phototherapy treatment and systemic treatments including corticosteroids, acitretin, cyclosporine and biological agents, and pregnant patients, and patients with a chronic systemic disease affecting the endocrine, cardiovascular, hematological, hepatic and renal systems, history of retinal disease, history of smoking and alcohol consumption, intraocular pressure $>21 \mathrm{~mm} \mathrm{Hg}$, any glaucomatous findings (such as glaucomatous optic disk changes, visual field defects), history of intraocular surgery or laser therapy, history of contact lens use, history of meibomian gland dysfunction, strabismus, history of ocular trauma, amblyopia, cataract, or presence of any ocular inflammatory disease were excluded. A control group was formed with healthy volunteers with no prior skin, eye or systemic diseases and with no history of any prior medication use.

\section{Dermatological examinations and laboratory parameters}

Detailed dermatological examinations of all patients with LP were performed by a dermatologist focusing on the involvement of skin, oral mucosa, the genital region and nails. Duration of the disease of all patients was recorded. Detailed laboratory examinations, including complete blood count, biochemical measurements, and hepatitis B and C status, were performed.

\section{Ophthalmologic examinations}

Only one eye (the right) per patient was selected. Each patient underwent a comprehensive eye examination including best corrected visual acuity, dilated fun- dus examination with 90-diopter (D) lens, AL, CCT, and Goldman applanation tonometry. The measurements of AL and CCT were conducted with an Echoscan US 500 device (Nidek Co. Ltd, Aichi, Japan). Additionally, Schirmer 1 and tear break-up time tests were performed. The Schirmer 1 test was performed by placing a Schirmer strip into the temporal inferior fornices. Topical anesthesia was applied. Results were read as the number of millimeters of wetting at $5 \mathrm{~min}$. The tear break-up time (TBUT) test was performed by instilling one drop of $2 \%$ sodium fluorescein dye into the eye without anesthesia. After asking the patient to blink three to four times, the tear film was observed using a biomicroscope with a blue cobalt filter with broad illumination. The time to the first break in the tear film complex after the last eye blink was measured.

The Ocular Surface Impairment Index (OSDI) questionnaire was performed in all patients. The OSDI questionnaire aims to evaluate the patient's complaints about dry eye in the last 2 weeks, and scales the severity of the disease by scoring the effects of these complaints on daily activities, environmental causes, duration and the severity of the complaints, along with the complaints of the patient [14]. RT and CT measurements were performed using spectral domain optical coherence tomography (SD-OCT) (Heidelberg Engineering, Heidelberg, Germany). The SD-OCT, which is a relatively new instrument for clinical in-vivo observation and retinal tissue followup evaluations, can execute repeated, high-resolution cross-sectional scans of the retina. The examination is more quantitative than an ophthalmoscopic examination, and even small changes in the retina can be detected. Computed tomography measurements were obtained using the enhanced depth imaging technique of SD-OCT. Ophthalmological examinations and OCT scans were performed by an experienced ophthalmologist at between 9:00 and 11:00 a.m. to avoid diurnal changes. The disc margin contour line was drawn manually at the inner edge of the scleral ring by determining 7 points. Computed tomography was measured as the perpendicular distance between the hyperreflective outer border of the retinal pigment epithelial layer (automatically detected by the instrument) and the sclerochoroidal interface, manually drawn. RT and CT measurements (one subfoveal, three temporal, three nasal) were obtained at $500 \mu \mathrm{m}$ intervals up to $1,500 \mu \mathrm{m}$ using the caliper system.

\section{Statistical analysis}

Normality of the data was tested with the ShapiroWilk test. The difference between the two groups was evaluated by Student's $t$-test. Pearson's correlation test was performed for analysis of the relationship between variables. A value of $p<0.05$ was considered statistically significant. 


\section{Results}

There were 33 patients (19 female and 14 male) in the LP group, with a mean age of $46.8 \pm 14$. 4 years (range: 20-70 years). The control group included 30 subjects (19 female and 11 male), with a mean age of $43.8 \pm 12.9$ years (range: $20-67$ years). There was no significant difference in terms of age and gender between the two groups $(p>0.05)$. The mean duration of LP was $27.1 \pm 46.2$ (range: 1-216 months) months. Among the LP patients, 1 (3\%) patient had a family history of LP; 15 (45.4\%) patients had oral mucosal involvement; 11 (33.3\%) patients had nail involvement; and 3 (9\%) patients had genital involvement. Oral mucosal involvement was of the reticular type in all patients, while clinically longitudinal grooving and ridging were present in the nails of all patients with nail involvement. All patients with genital involvement were male, and penile annular plaque was seen in all of them.

There was no significant difference between the groups in terms of IOP, CCT and AL $(p>0.05)$. The mean Schirmer 1 values were $9.9 \pm 6.8 \mathrm{~mm}$ for patients and 17.2 $\pm 9.5 \mathrm{~mm}$ for controls $(p=0.001)$. The mean TBUT values were $7.0 \pm 2.5 \mathrm{~s}$ in the LP group and $14.8 \pm 7.1 \mathrm{~s}$ in the control group ( $p=0.001)$. The mean OSDI score values were $36.9 \pm 21.9$ in patients and $12 \pm 5.3$ in controls $(p=0.001)$. The demographic and clinical characteristics of the study sample and the statistical significance of the differences between the study groups are shown in Table 1.

There was no significant difference for mean RT when the two groups were compared $(p>0.05)$. The mean CT was found to be significantly thinner in the patients compared with the controls ( $p=0.001)$. Changes in both the mean RT and the mean CT are presented in Table 2.

There was a moderate negative correlation between oral mucosal involvement and Schirmer 1, TBUT, and subfoveal CT, while there was a moderate positive correlation with OSDI $(r=-0.345, r=-0.392, r=-0.467$, $r=0.345$, respectively; $p<0.05$ in each group). There was a moderate negative correlation between duration of disease and Schirmer 1, TBUT, and subfoveal CT, while
Table 1. Demographic and clinical characteristics of the study groups

\begin{tabular}{lccc}
\hline Parameters & LP & Control & $P$-value \\
\hline Age $[$ years] & $46.8 \pm 14.4$ & $43.8 \pm 12.9$ & 0.393 \\
\hline IOP $[\mathrm{mm} \mathrm{Hg}]$ & $15.4 \pm 2.5$ & $14.3 \pm 2.6$ & 0.096 \\
\hline CCT $[\mu \mathrm{m}]$ & $546.7 \pm 33.8$ & $541.1 \pm 23.4$ & 0.456 \\
\hline AL $[\mathrm{mm}]:$ & $22.8 \pm 0.9$ & $22.9 \pm 0.8$ & 0.488 \\
\hline Schirmer 1 $[\mathrm{mm}]$ & $9.9 \pm 6.8$ & $17.2 \pm 9.5$ & 0.001 \\
\hline TBUT $[\mathrm{s}]$ & $7.0 \pm 2.5$ & $14.8 \pm 7.1$ & 0.001 \\
\hline OSDI & $36.9 \pm 21.9$ & $12 \pm 5.3$ & 0.001 \\
\hline
\end{tabular}

IOP - intraocular pressure, CCT - central corneal thickness, AL - axial length, BUT - tear break-up time, OSDI - ocular surface impairment index.

there was a moderate positive correlation with OSDI $(r=-0.426, r=-0.555, r=-0.637, r=0.717$, respectively; $p<0.05$ in each group). There was no statistically significant correlation between duration of disease and CCT or foveal retinal thickness (FRT) $(r=0.003, r=-0.031$; $p=0.984, p=0.809$, respectively). There was no statistically significant correlation between oral mucosal involvement and CCT or FRT $(r=0.124, r=-0.074$; $p=0.333, p=0.563$, respectively) (Table 3 ).

\section{Discussion}

The results of this prospective case-control study indicate that there are significant differences in the Schirmer 1 test, TBUT, OSDI questionnaire, and CT between patients with LP and healthy controls. In the current study, CT was significantly thinner in LP patients than in the control group. Duration of LP and oral mucosal involvement also may be an important determinant of ocular involvement.

Lichen planus is a disease with an incompletely understood etiology that affects oral and genital mucosa [15]. Fewer than 20 cases with LP and eyelid involvement have been reported [4]. However, no eyelid involvement was diagnosed in any case with LP in our study. Ocular LP

Table 2. Distribution of mean choroidal and retinal thickness between the groups

\begin{tabular}{|c|c|c|c|c|c|c|}
\hline \multirow[t]{2}{*}{ Location } & \multicolumn{3}{|c|}{ Choroidal thickness $[\mu \mathrm{m}]$} & \multicolumn{3}{|c|}{ Retinal thickness $[\mu \mathrm{m}]$} \\
\hline & $\operatorname{LP}(n=33)$ & Control $(n=30)$ & $P$-value & $\operatorname{LP}(n=33)$ & Control $(n=30)$ & $P$-value \\
\hline Foveal & $230.2 \pm 40.6$ & $305.9 \pm 45.8$ & 0.001 & $221.2 \pm 19.4$ & $221.5 \pm 23.2$ & 0.966 \\
\hline Temporal $500 \mu \mathrm{m}$ & $229.9 \pm 43$ & $303 \pm 41.4$ & 0.001 & $280.5 \pm 34$ & $274.3 \pm 29.3$ & 0.443 \\
\hline Temporal $1000 \mu \mathrm{m}$ & $227.6 \pm 49.7$ & $294.6 \pm 40.5$ & 0.001 & $326.7 \pm 22.6$ & $327.4 \pm 17.1$ & 0.895 \\
\hline Temporal $1500 \mu \mathrm{m}$ & $221 \pm 44.9$ & $293.8 \pm 44.3$ & 0.001 & $325.5 \pm 17$ & $329.7 \pm 14.4$ & 0.308 \\
\hline Nasal $500 \mu \mathrm{m}$ & $230.8 \pm 39.2$ & $303.9 \pm 42.5$ & 0.001 & $301.8 \pm 28.4$ & $300.2 \pm 26.2$ & 0.813 \\
\hline Nasal $1000 \mu \mathrm{m}$ & $227 \pm 39.8$ & $291.5 \pm 39.9$ & 0.001 & $344.8 \pm 28.7$ & $346.4 \pm 15.4$ & 0.789 \\
\hline Nasal $1500 \mu \mathrm{m}$ & $217.7 \pm 45.9$ & $272.1 \pm 45$ & 0.001 & $348.3 \pm 17.3$ & $347.4 \pm 14.9$ & 0.809 \\
\hline
\end{tabular}

LP-lichen planus. 
Table 3. Correlation analyses between duration of disease, oral mucosal involvement and Schirmer 1, TBUT, OSDI, CCT, FRT, SFCT

\begin{tabular}{|c|c|c|c|c|c|c|}
\hline Parameter & Schirmer 1 & TBUT & OSDI & CCT & FRT & SFCT \\
\hline \multicolumn{7}{|c|}{ Oral mucosal involvement: } \\
\hline$r$ & -0.345 & -0.392 & 0.345 & 0.124 & -0.074 & -0.467 \\
\hline$p$ & 0.006 & 0.001 & 0.006 & 0.333 & 0.563 & 0.001 \\
\hline \multicolumn{7}{|l|}{ DD: } \\
\hline$r$ & -0.426 & -0.555 & 0.717 & 0.003 & -0.031 & -0.637 \\
\hline$p$ & 0.001 & 0.001 & 0.001 & 0.984 & 0.809 & 0.001 \\
\hline
\end{tabular}

DD - duration of disease, TBUT - tear break-up time, OSDI - ocular surface impairment index, CCT - central corneal thickness, FRT-foveal retinal thickness, SFCT - subfoveal choroidal thickness.

may be manifested with canalicular pathologies $[5,16]$. Eye involvement is rare in LP; however, cicatrizing conjunctivitis is one of the well-known complications of the disease [2, 3]. Brewer et al. [2] reported that the disease was controlled well by topical or systemic immunosuppressive drugs in 8 out of 11 patients with LP with cicatrizing conjunctivitis. Thorne et al. [3] reported that 5 out of 6 patients with LP with cicatrizing conjunctivitis had oral mucosal and gingival involvement and 1 patient had skin involvement of LP. The general health condition of patients with ocular LP was frequently good; however, accompanying thymoma and lymphoma were detected in 2 patients with LP and cicatrizing conjunctivitis, and this condition was suggested to be a paraneoplastic phenomenon [15].

Lichen planus may affect the ocular surface due to the conjunctival involvement. Rarely, conjunctival inflammation and subepithelial scarring may occur secondary to conjunctival involvement and keratitis, and keratoconjunctivitis may develop due to corneal involvement [7]. There is only one case-control study evaluating the ophthalmological findings in LP. Thus, there is a paucity of data available. The only case-control study that has evaluated the ophthalmological findings in LP is that of Şanli et al. [7], who evaluated the ophthalmological findings of 20 cases with LP and 24 controls using conjunctival impression cytology, the Schirmer test, break-up time, fluorescein and lissamine green scoring tests. The Schirmer value was found to be significantly lower and lissamine green staining scores were significantly higher in patients with LP compared to the control group. Impression cytology, on the other hand, revealed that conjunctival epithelial goblet cell loss was significantly higher in patients with LP compared to the control group, and they suggested that LP affected the tear film and the health of the ocular surface. We also found lower Schirmer 1 test values and TBUT in patients with LP than the controls in the present study, but we did not perform impression cytology. A negative correlation was present between the Schirmer 1 test and both duration of the disease and oral mucosal involvement. A negative correlation was also found between the TBUT and both duration of the disease and oral mucosal involvement. A positive correlation was also detected between the OSDI score and both duration of the disease and oral mucosal involvement. These findings suggest that the anterior segment of the eye is affected in patients with LP. It seems likely that the duration of disease and oral mucosal involvement may be poor prognostic factors in terms of the severity of the ocular involvement.

It is known that metabolic and cardiovascular risk factors are more common among patients with LP compared to the normal population [1]. Increased risk of metabolic and cardiovascular diseases was suggested to develop due to the long-term inflammation [17]. Ataş et al. [18] evaluated the systemic inflammation of LP with the neutrophil-lymphocyte rate (NLR) and found that NLR was higher in the LP group compared to the control group. Their findings suggest that LP is not only an inflammatory disease confined to the skin but also is a systemic inflammatory disease. Studies were conducted aimed at evaluation of eye involvement in psoriasis, another skin disease characterized by systemic inflammation. Aragona et al. [19] demonstrated that ocular surface tests were impaired significantly in patients with psoriasis compared to the healthy control group in their study. However, there was no significant difference in the ocular surface test results between the patients with and without psoriatic arthritis. On the other hand, Celik et al. [20] suggested that psoriasis affected the corneal biomechanical properties. Demirci et al. [21] found out that osmolarity values, OSDI and Oxford scale scores were significantly higher and the tear film break-up time was significantly shorter in patients with psoriasis compared to the healthy controls. Some researchers evaluated the posterior segment structures of the eye in psoriasis, which had similar aspects with LP in the pathogenesis since it also involved systemic inflammation. However, the results of the clinical trials have been inconsistent. Kılıç et al. [22] found a positive correlation between the duration of the disease and $\mathrm{CT}$ in patients with psoriasis. In addition, they found no significant difference between the patients and controls in terms of CT. Türkcü et al. [23], on the other hand, reported that $C T$ in patients with 
psoriasis was significantly higher compared to the control group. Ersan et al. [13] described choroidal changes in patients with psoriasis without accompanying macular changes. The previous studies reported that CT may increase or decrease in chronic inflammatory diseases other than psoriasis $[24,25]$. To our knowledge, this is the first study in the literature that has evaluated the posterior segment of the eye among patients with LP. We found that the choroid in LP patients was thinner than that in the controls and a negative correlation was present between the duration of the disease, oral mucosal involvement and CT. However, no significant difference was observed between the LP and control groups for mean RT.

This study has some limitations. First, our study had a relatively small sample size. Second, we did no scoring for the severity of the disease in patients with LP. Therefore, we could not evaluate the association between the severity of the disease and ocular findings. More comprehensive studies with larger patient groups that evaluate the association of LP, the severity of the disease and ocular findings are required.

\section{Conclusions}

Lichen planus is not a disease affecting only the skin. It is notable that LP may affect many organs including the eye by causing systemic inflammation. This study revealed that LP influences both the anterior segment of the eye and the CT. Lichen planus should be kept in mind in the differential diagnosis of dry eye disease. The results of this study should be confirmed with further studies.

\section{Conflict of interest}

The authors declare no conflict of interest.

\section{References}

1. Ozlu E, Karadag AS, Toprak AE, et al. Evaluation of cardiovascular risk factors, haematological and biochemical parameters, and serum endocan levels in patients with lichen planus. Dermatology 2016; 232: 438-43.

2. Brewer JD, Ekdawi NS, Torgerson RR, et al. Lichen planus and cicatricial conjunctivitis: disease course and response to therapy of 11 patients. J Eur Acad Dermatol Venereol 2011; 25: 100-4.

3. Thorne JE, Jabs DA, Nikolskaia OV, et al. Lichen planus and cicatrizing conjunctivitis: characterization of five cases. Am J Ophthalmol 2003; 136: 239-43.

4. Huang YY, Wang CM, Potenziani S, Hsu S. Lichen planus of the eyelids: a case report and review of the literature. Dermatol Online J 2017; 23: pii: 13030/qt1c04h08s.

5. Webber NK, Setterfield JF, Lewis FM, Neill SM. Lacrimal canalicular duct scarring in patients with lichen planus. Arch Dermatol 2012; 148: 224-7.

6. Boyce AE, Marshman G, Mills RA. Erosive mucosal lichen planus and secondary epiphora responding to systemic cyclosporin A treatment. Australas I Dermatol 2009; 50: 190-3.
7. Şanli B, Çetin EN, Bir F, et al. Conjunctival impression cytology, ocular surface and tear-film changes in patients with lichen planus. Clin Exp Dermatol 2012; 37: 341-5.

8. Akkurt ZM, Gümüş H, Aktürk A, et al. Evaluation of orbital arteries with colour Doppler ultrasonography in patients with psoriasis. Clin Exp Dermatol 2015; 40: 507-12.

9. Kilic B, Dogan U, Parlak AH, et al. Ocular findings in patients with psoriasis. Int J Dermatol 2013; 52: 554-9.

10. Chandran NS, Greaves M, Gao F, et al. Psoriasis and the eye: prevalence of eye disease in Singaporean Asian patients with psoriasis. J Dermatol 2007; 34: 805-10.

11. Campanati A, Neri P, Giuliodori K, et al. Psoriasis beyond the skin surface: a pilot study on the ocular involvement. Int Ophthalmol 2015; 35: 331-40.

12. Mrejen S, Spaide RF. Optical coherence tomography: imaging of the choroid and beyond. Surv Ophthalmol 2013; 58: 387-429.

13. Ersan I, Kilic S, Arikan S, et al. Evaluation of macular ganglion cell-inner plexiform layer and choroid in psoriasis patients using enhanced depth imaging spectral domain optical coherence tomography. Ocul Immunol Inflamm 2017; 25: 520-4.

14. Cömez AT, Tufan HA, Kocabıyık O, et al. Effects of lubricating agents with different osmolalities on tear osmolarity and other tear function tests in patients with dry eye. Curr Eye Res 2013; 38: 1095-103.

15. Webster GF, Durrani K, Suchecki J. Ocular rosacea, psoriasis, and lichen planus. Clin Dermatol 2016; 34: 146-50.

16. Durrani OM, Verity DH, Meligonis G, Rose GE. Bicanalicular obstruction in lichen planus: a characteristic pattern of disease. Ophthalmology 2008; 115: 386-9.

17. Saleh N, Samir N, Megahed H, Farid E. Homocysteine and other cardiovascular risk factors in patients with lichen planus. J Eur Acad Dermatol Venereol 2014; 28: 1507-13.

18. Ataş H, Cemil BÇ, Kurmuş GI, Gönül M. Assessment of systemic inflammation with neutrophil-lymphocyte ratio in lichen planus. Adv Dermatol Allergol 2016; 33: 188-92.

19. Aragona E, Rania L, Postorino El, et al. Tear film and ocular surface assessment in psoriasis. Br J Ophthalmol 2018; 102: 302-8.

20.Celik U, Aykut V, Celik B, et al. A comparison of corneal biomechanical properties in patients with psoriasis and healthy subjects. Eye Contact Lens 2015; 41: 127-9.

21. Demirci G, Erdur SK, Aydin R, et al. Tear osmolarity and ocular surface parameters in patients with psoriasis. Arq Bras Oftalmol 2017; 80: 1-3.

22. Kılıç R, Kurt A, Acer E, et al. Choroidal thickness in psoriasis. Int Ophthalmol 2017; 37: 173-7.

23. Türkcü FM, Şahin A, Yüksel H, et al. Evaluation of choroidal thickness in psoriasis using optical coherence tomography. Int Ophthalmol 2016; 36: 851-4.

24. Coskun E, Gurler B, Pehlivan Y, et al. Enhanced depth imaging optical coherence tomography findings in Behçet disease. Ocul Immunol Inflamm 2013; 21: 440-5.

25. Kola M, Kalkisim A, Karkucak M, et al. Evaluation of choroidal thickness in ankylosing spondylitis using optical coherence tomography. Ocul Immunol Inflamm 2014; 22: 434-8. 